\title{
Correlations in orbital angular momentum of spatially entangled paired photons generated in parametric downconversion
}

\author{
Clara I. Osorio ${ }^{1}$, Gabriel Molina-Terriza ${ }^{1,2}$ and Juan P. Torres ${ }^{1,3}$ \\ ${ }^{1}$ ICFO-Institut de Ciencies Fotoniques, Mediterranean Technology Park, 08860 Castelldefels (Barcelona) Spain \\ ${ }^{2}$ ICREA-Institucio Catalana de Recerca $i$ Estudis Avancats, 08010 Barcelona, Spain and \\ 3 Dept. Signal Theory and Communications, Universitat Politecnica de Catalunya, Campus Nord, 08034 Barcelona Spain ${ }^{*}$
}

\begin{abstract}
What are the orbital angular momentum correlations between spatially entangled photon pairs generated in spontaneous parametric down-conversion? We show that the answer to this question can be given in two alternative, although complementary, ways. The answer posed in this letter explains satisfactorily the seemingly contradictory results obtained in different experiments, and theoretical approaches.
\end{abstract}

PACS numbers: 03.67.Mn, 42.50.Dv, 42.65.Lm

Within the paraxial quantum optics regime, the orbital angular momentum (OAM) provides an useful description of the spatial degree of freedom of photons. Photons whose spatial waveform contains an azimuthal phase dependence of the form $\sim \exp (i m \varphi)$, carry an OAM of $m \hbar$ per photon [1]. Photons with diverse spatial waveforms can be easily generated, detected, and controlled. Therefore, the OAM offers a physical resource where to explore deeper quantum features not present in the 2dimensional Hilbert space addressed when using the polarization [2, 3]. Indeed, it allows to readily tailor the number of effective dimensions of the Hilbert space [4].

During the last few years, several quantum features based on the capacity of the OAM of photons to go beyond a 2-D Hilbert space have been demonstrated (see 5] and references inside) using spontaneous parametric down-conversion (SPDC). These include the demonstration of the violation of bipartite, three dimensional Bell inequalities [6], the implementation of the so called quantum coin tossing protocol with qutrits [], and the generation of quantum states in ultra-high dimensional spaces [8].

All of these experiments make use of the existence of specific quantum OAM correlations between the two entangled photons generated in the SPDC process. Several experiments [2, 9, 10, 11] seems to support the validity of the selection rule $m_{p}=m_{1}+m_{2}$, where $m_{p} \hbar$ is the OAM per photon of the classical pump beam, and $m_{1}$ and $m_{2}$ are the winding numbers of the modes into which the quantum state of the signal and idler photons are projected, respectively. Some other experiments [12, 13, 14], while not directly measuring the OAM of the downconverted photons, demonstrate the existence of ellipticity of the spatial waveform, which should make possible the detection of photons with $m_{p} \neq m_{1}+m_{2}$. Under some restrictive conditions, the selection rule $m_{p}=m_{1}+m_{2}$ can be derived from first principles [4, 15, 16], although, as it will be shown below, the same rule address differ-

*Electronic address: juan.perez@icfo.es ent physical quantities. The presence of Poynting vector walk-off can also strongly modify OAM correlations [17].

All this raises the question of what are the OAM correlations between the downconverted photons generated in SPDC, i.e., under which conditions the OAM of the entangled photons fulfill the selection rule $m_{p}=m_{1}+m_{2}$. Here we show that this question can be formulated in two complementary scenarios, so that in each scenario the sough-after OAM correlations can be different. The existence of previous apparently contradictory results is due to the fact that the sought-after OAM correlations are different.

In one scenario, the spatial properties of all the pairs of photons generated are considered, therefore the global mode function is obtained adding coherently all such possibilities. In another scenario, which is relevant for current experimental applications, a small section of the full down-conversion cone is considered. Only certain probability amplitudes are now considered. Under these conditions, the non-collinear SPDC geometry and the presence of spatial walk-off can greatly modify the OAM correlations observed.

We consider a nonlinear crystal of length $L$, illuminated by a monochromatic laser pump beam propagating in the $z$ direction, with frequency $\omega_{p}$. The spatial shape of the pump beam at the input face of the nonlinear crystal $(z=0)$, in the transverse wavevector domain, writes $E_{p}^{+}(\overline{\mathbf{p}})=E_{0}\left(\bar{p}_{x}+i \bar{p}_{y}\right)^{m_{p}} \exp \left(-|\overline{\mathbf{p}}|^{2} w_{0}^{2} / 4\right)$, which corresponds to a beam which carries an OAM of $m_{p} \hbar$ per photon. $E_{0}$ is a normalizing constant, $\overline{\mathbf{p}}=\left(\bar{p}_{x}, \bar{p}_{y}\right)$ is the transverse wavevector and $w_{0}$ is the beam width. The signal and idler photons are assumed to be monochromatic, with $\omega_{s}=\omega_{i}=\omega_{p} / 2$, where $\omega_{s, i}$ are the frequencies of the signal and idler photons. This is justified by the use of narrow-band interference filters in front of the detectors.

The photons are known to be generated into cones, whose shape is determined by the phase matching conditions inside the crystal. For the sake of clarity, let us consider first noncritical, i.e. negligible walk-off, noncollinear SPDC in a periodically poled nonlinear crystal. The angle of the down conversion cone is assumed to be 
small, so that the polarization [18] and refractive index do not show noticeable changes with the direction of propagation. Similarly, the nonlinear coefficient is assumed to be constant along the down-conversion cone.

The SPDC process can be described in the interaction picture by an effective Hamiltonian given by [19] $H_{I}=\epsilon_{0} \int_{V} d V \chi^{(2)} E_{p}^{+} E_{s}^{-} E_{i}^{-}+$c.c, where $E_{s}^{-}(\mathbf{x}, z, t) \propto$ $\int d K_{s} d \mathbf{P} \exp \left(-i \mathbf{P} \cdot \mathbf{x}-i K_{s} z+i \omega_{s} t\right) a^{\dagger}\left(K_{s}, \mathbf{P}\right)$ refers to the negative-frequency part of the signal electric field operators. Similarly for the idler photon. The two-photon quantum state $|\Psi\rangle$ at the output face of the nonlinear crystal, within the first order perturbation theory, can be written as $|\Psi\rangle=\int d \mathbf{P} d \mathbf{Q} \Phi(\mathbf{P}, \mathbf{Q}) a_{s}^{\dagger}(\mathbf{P}) a_{i}^{\dagger}(\mathbf{Q})|0,0\rangle$, where $\mathbf{P}$ and $\mathbf{Q}$ are the transverse wavevector for the signal and the idler respectively, and the mode function $\Phi$ is given by

$$
\begin{aligned}
& \Phi(\mathbf{P}, \mathbf{Q})=E_{p}(\mathbf{P}+\mathbf{Q}) \operatorname{sinc}\left(\frac{\Delta_{k} L}{2}\right) \\
& \exp \left\{i \frac{\Delta_{k} L}{2}+i\left[K_{s}(\mathbf{P})+K_{i}(\mathbf{Q})\right] L\right\}
\end{aligned}
$$

where $\Delta_{k}=K_{p}(\mathbf{P}+\mathbf{Q})-K_{s}(\mathbf{P})-K_{i}(\mathbf{Q})$, the wavevectors $(j=s, i, p)$ write $K_{j}(\mathbf{P})=\left[\left(\omega_{j} n_{j} / c\right)^{2}-|\mathbf{P}|^{2}\right]^{1 / 2}$, depends on the modulus of the corresponding transverse wavevectors, and $n_{j}$ are the corresponding refractive index. The mode function of the biphoton in the spatial domain $\left(\mathbf{x}_{1}, \mathbf{x}_{2}\right)$ is the spatial Fourier transform of the mode function given by Eq. (1).

We can write $|\mathbf{P}+\mathbf{Q}|^{2}=\rho_{s}^{2}+\rho_{i}^{2}+2 \rho_{s} \rho_{i} \cos \left(\varphi_{s}-\varphi_{i}\right)$, where $\rho_{s}=|\mathbf{P}|$, and $\varphi_{s}=\tan ^{-1} P_{y} / P_{x}$ are the modulus and phase of the transverse wave vector $\mathbf{P}$ in cylindrical coordinates. For the idler photon we have, similarly, $\rho_{i}=|\mathbf{Q}|$ and $\varphi_{i}=\tan ^{-1} Q_{y} / Q_{x}$. One can write $\operatorname{sinc}\left(\Delta_{k} L / 2\right) \exp \left(i \Delta_{k} L / 2\right)=$ $\sum_{l=-\infty}^{\infty} \mathcal{H}_{l}\left(\rho_{s}, \rho_{i}\right) \exp \left\{i l\left(\varphi_{s}-\varphi_{i}\right)\right\} . \quad K_{s, i}$ depends on the moduli $\rho_{s, i}$, respectively. The pump beam can also be written as

$$
\begin{aligned}
& E_{p}=E_{0} \exp \left\{-\frac{\left[\rho_{s}^{2}+\rho_{i}^{2}+\rho_{s} \rho_{i} \cos \left(\varphi_{s}-\varphi_{i}\right)\right] w_{0}^{2}}{4}\right\} \\
& \times \sum_{l=0}^{m_{p}}\left(\begin{array}{c}
m_{p} \\
l
\end{array}\right) \rho_{s}^{l} \rho_{i}^{m_{p}-l} \exp \left\{i l \varphi_{s}+i\left(m_{p}-l\right) \varphi_{i}\right\}
\end{aligned}
$$

Therefore, the mode function given by Eq. (11) can be written as

$$
\Phi(\mathbf{P}, \mathbf{Q})=\sum_{m=-\infty}^{\infty} \mathcal{G}_{m}\left(\rho_{s}, \rho_{i}\right) \exp \left[i m \varphi_{s}+i\left(m_{p}-m\right) \varphi_{i}\right]
$$

The main conclusion to be drawn from Eq. (3) is that, if polarization, refractive index and nonlinear coefficient show negligible azimuthal variations around the downconversion cone, the OAM correlations of the spatial waveform of the biphoton state fulfill $m_{p}=m_{1}+m_{2}$ [15]. Importantly, this result requires considering the whole
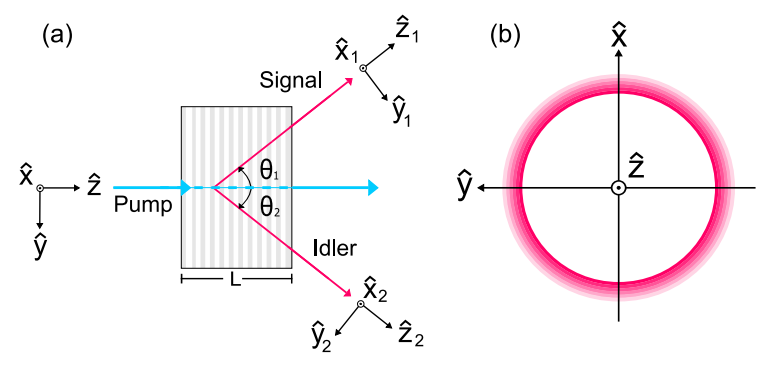

FIG. 1: Schematic diagram of a non-collinear SPDC. (a) Top view of the non-collinear configuration. (b) The downconversion cone.

spatial waveform of the downconverted photons, i.e. the full down-conversion cone. Notwithstanding, these are not the OAM correlations that typical quantum information experiments based on spatial entanglement measure. An experiment aimed at detecting the global OAM of the downconverted photons is a significant experimental challenge that it is yet to be solved.

All relevant experiments reported to date detect only a small section of the full down conversion cone. In other words, the wave vectors of the signal and idler photons belong to a narrow bundle around the corresponding central wavevectors, i.e. $\mathbf{P}=\mathbf{P}_{0}+\Delta \mathbf{P}$ and $\mathbf{Q}=\mathbf{Q}_{0}+\Delta \mathbf{Q}$. As shown in Fig. 1, the signal photon propagate along the direction $\hat{z}_{1}$ with longitudinal wavevector $k_{s}(\mathbf{p})=\left[\left(w_{s} n_{s} / c\right) 2-|\mathbf{p}|^{2}\right]^{1 / 2}$, and transverse wavevector $\mathbf{p}=\left(p_{x}, p_{y}\right)$, so that $\Delta P_{x}=p_{x}$ and $\Delta P_{y}=\cos \theta_{1} p_{y}-\sin \theta_{1} k_{s}$. And similarly for the idler photon, which propagates in the direction $\hat{z}_{2}$ with longitudinal wavevector $k_{i}(\mathbf{q})$, and transverse wavevector $\mathbf{q}$, so that $\Delta Q_{x}=q_{x}$ and $\Delta Q_{y}=\cos \theta_{2} q_{y}-\sin \theta_{2} k_{i}$. We restrict ourselves to the case $\theta_{1}=-\theta_{2}=\theta$.

The quantum state of the biphoton at $z_{1}=L / \cos \theta$ can be written as $|\psi\rangle=\int d \mathbf{p} d \mathbf{q} \Phi(\mathbf{p}, \mathbf{q}) a_{s}^{+}(\mathbf{p}) a_{i}^{+}(\mathbf{q})|0,0\rangle$, where the mode function writes [20]

$$
\begin{aligned}
& \Phi(\mathbf{p}, \mathbf{q})=E_{p}\left(p_{x}+q_{x}, \delta_{0}\right) \operatorname{sinc}\left(\frac{\delta_{k} L}{2}\right) \\
& \exp \left\{i \frac{\delta_{k} L}{2}+i\left[k_{s}(\mathbf{p})+k_{i}(\mathbf{q})\right] \frac{L}{\cos \theta}\right\}
\end{aligned}
$$

where $\delta_{k}=k_{p}-\left(k_{s}+k_{i}\right) \cos \theta-\left(p_{y}-q_{y}\right) \sin \theta$ and $\delta_{0}=$ $\left(p_{y}+q_{y}\right) \cos \theta-\left(k_{s}+k_{i}\right) \sin \theta$.

The mode function given by Eq. (4) shows ellipticity in the $(\mathbf{p}, \mathbf{q})$ domain, as has been demonstrated experimentally [12, 14]. An increasing degree of ellipticity of the spatial mode function enhances the quantum probability amplitude of paired photons with $m_{p} \neq m_{1}+m_{2}$. To get further insight in the nature of the OAM correlations, let us consider that the idler photon is projected into a gaussian mode $\left(m_{2}=0\right)$, so that the quantum state of the signal photon is described by the reduced mode function $\Phi_{s}(\mathbf{p}) \propto \int d \mathbf{q} \Phi(\mathbf{p}, \mathbf{q}) \exp \left(-|\mathbf{q}|^{2} w_{1}^{2} / 4\right)$, where $w_{1}$ is the beam width of the idler mode. To elucidate the OAM 


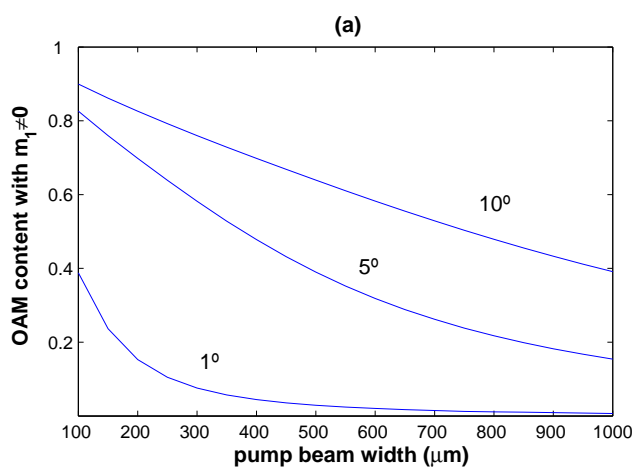

(b)

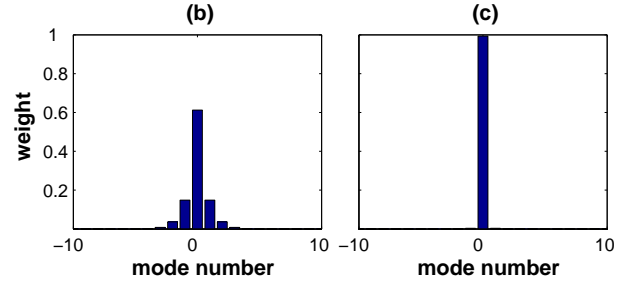

FIG. 2: Orbital angular momentum of the signal photon in non-collinear SPDC in a $L=10 \mathrm{~mm}$ long PPKTP crystal. The pump beam is gaussian $\left(m_{p}=0\right)$. Both photons traverse 2 -f systems. The idler photon is detected with $\mathbf{q}=\mathbf{0}$. (a) Total weight of the OAM modes with $m_{1} \neq 0$, as a function of the pump beam width $\left(w_{0}\right)$. The label designate the noncollinear angle. (b) and (c) OAM mode distribution for $\varphi=$ $1^{\circ}$ for two values of the pump beam width: $w_{0}=100 \mu \mathrm{m}$, and $w_{0}=1000 \mu \mathrm{m}$.

content of the signal photon, one has to project the spatial mode function into spiral harmonics $\exp (\operatorname{im} \varphi)$. The weights for each $m$ of such decomposition gives us the sought-after OAM decomposition [3].

Fig. 2(a) shows the total weight corresponding to OAM modes with $m_{1} \neq 0$, which is a measure of the degree of violation of the selection rule $m_{p}=m_{1}+m_{2}$, for different values of the non-collinear angle and the pump beam width. Notice that the larger the non-collinear angle $\theta$, and the smaller the pump beam width $w_{0}$, the larger is the probability to detect signal photons with $m_{1} \neq 0$. Figs. 2(b) and 2(c) show two OAM distributions for $\theta=1^{\circ}$, one which shows an OAM distribution with modes with $m_{1} \neq 0$, while the other shows a single peak for $m_{1}=0$.

The strength of the violation of the selection rule $m_{p}=m_{1}+m_{2}$ can be quantified through the noncollinear length [17] $L_{n c}=w_{0} / \sin \varphi$. If the crystal length is much smaller than the non-collinear length $\left(L \ll L_{n c}\right)$, the ellipticity of the mode function is small, and thus the selection rule $m_{p}=m_{1}+m_{2}$ is fulfilled. This turns out to be the case of nearly all of the experiments that make use of the OAM of photons [2, 6, 6, 8, 9, 10, 11]. Typical non-collinear angles and crystal lengths used in these experiments are $\theta \simeq 1-2^{\circ}$ and $L \simeq 1-5 \mathrm{~mm}$. For $w_{0}=500 \mu \mathrm{m}$, one obtains $L_{n c} \simeq 15-30 \mathrm{~mm}$, so that $L<L_{n c}$. On the contrary, if $L \geq L_{n c}$, strong departures from the selection rule $m_{p}=m_{1}+m_{2}$ are expected. This is the experimental configuration in [12, 13, 14], due to the use of a highly focused pump beam or longer crystals. For $w_{0}=90 \mu \mathrm{m}$ and $\theta=2^{\circ}$, one has $L_{n c} \simeq 2.5 \mathrm{~mm}$.

According to Eq. (3), the OAM of the spatially entangled photons fulfill the relationship $m_{p}=m_{1}+m_{2}$, while Fig. 2 shows that strong departures from this selection rule can be observed if highly focused pump beams, highly non-collinear configurations or longer nonlinear crystals are used. Actually, we are describing the same quantum process in two complementary scenarios. Fig. 2 would give us the OAM correlations measured in typical experiments that use the OAM as physical resource for quantum information, thus it is relevant for experimental configurations currently used. In this scenario, the fulfillment of the condition $m_{p}=m_{1}+m_{2}$ depends on the pump beam width and the non-collinear angle, as dictated by the interplay between the non-collinear and crystal lengths.

On the other hand, Eq. (3) corresponds to a global view of the SPDC process, where the full downconversion cone is considered. The question of angular momentum conservation balance in SPDC requires the simultaneous consideration of the angular momentum of the electronic spins and orbitals, the crystalline structure of the nonlinear crystal and of the electromagnetic field [21]. The analysis presented here might be an important step towards clarifying how angular momentum is effectively conserved, since to evaluate conservation laws, one should take into account all probabilities amplitudes that contribute to the quantum process.

Another important effect that might modify the OAM correlations is the presence of Poynting vector walk-off in some, or all, of the interacting waves. In type I and type II SPDC configurations, some of the interacting waves are extraordinary waves, thus show Poynting vector walk-off. For the sake of simplicity, let us consider a type I configuration, where only the pump beam presents spatial walk-off. An initially gaussian pump beam, at each position $z$ inside the nonlinear crystal can be decomposed into spiral harmonics as

$$
\begin{aligned}
& E_{p}\left(\rho_{\mathbf{p}}, z\right)=E_{0} \exp \left\{-\rho_{p}^{2}\left(\frac{w_{0}^{2}}{4}+i \frac{z}{2 k_{p}^{0}}\right)\right\} \\
& \times \sum_{n=-\infty}^{\infty} J_{n}\left(z \rho_{p} \tan \rho_{0}\right) \exp \left\{i n \varphi_{p}\right\}
\end{aligned}
$$

where $\rho_{0}$ is the spatial walk-off angle, $\varphi_{p}$ the azimuthal angle in cylindrical coordinates and $J_{n}$ are Bessel functions of the first kind, and $k_{p}^{0}$ is the longitudinal wavevector. As shown in Fig. 3, the OAM distribution of the pump beam increases its width with distance $z$. A gaussian beam incident from air into the nonlinear crystal is no longer a gaussian beam, i.e. the pump beam is no longer a wave with $m_{p}=0$.

This effect becomes noticeable only if $L>L_{w}$, where the walk length writes $L_{w}=w_{0} / \tan \rho_{0}$. Fig. 3(a) shows the initial OAM distribution of a gaussian beam, which corresponds to a single peak with $m_{p}=0$. Figs $3(\mathrm{~b})$ and 

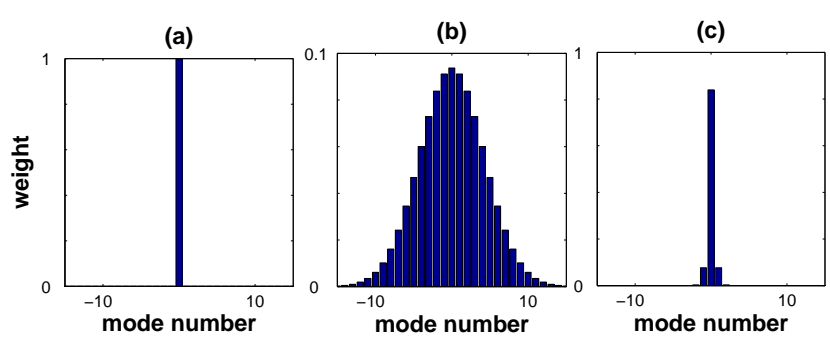

FIG. 3: Orbital angular momentum distribution of the pump beam in different positions inside the nonlinear crystal. (a) $z=0$; (b) $z=5 \mathrm{~mm}$ and $w_{0}=100 \mu \mathrm{m}$ and (c) $z=5 \mathrm{~mm}$ and $w_{0}=1 \mathrm{~mm}$. The walk-off angle is $\rho_{0}=5^{\circ}$.

3 (c) show the OAM distribution at $L=5 \mathrm{~mm}$ for a walkoff angle of $\rho_{0}=5^{\circ}$. For highly focused pump beams, the walk off length can become smaller than the crystal length, thus the OAM distribution no longer shows a single peak, as shown in Fig. 3(b). In this case, $L_{w} \simeq 1$ $\mathrm{mm}$. The walk off effect might be negligible for larger beam widths, as one can see in Fig. 3(c). Now, one obtains $L_{w} \simeq 12 \mathrm{~mm}$.

In the walking SPDC consider, the spatial waveform of the two-photon can be written as

$$
\begin{aligned}
& \Phi(\mathbf{P}, \mathbf{Q})=E_{p}(\mathbf{P}+\mathbf{Q}) \operatorname{sinc}\left(\Delta_{k} L / 2\right) \\
& \exp \left\{i \frac{\Delta_{k} L}{2}+i\left[K_{s}(\mathbf{P})+K_{i}(\mathbf{Q})\right] L\right\}
\end{aligned}
$$

where $\Delta_{k}=k_{p}(\mathbf{P}+\mathbf{Q})+\left(P_{x}+Q_{x}\right) \tan \rho_{0}-k_{s}(\mathbf{P})-$ $k_{i}(\mathbf{Q})$. The phase matching function no longer depends on $\varphi_{s}-\varphi_{i}$ due to the presence of the spatial walk-off.

The mode function of the two-photon state is a coherent sum of all the partial amplitudes due to the possible generation of a pair of photons at position $z$ inside the crystal 222]. Since the beam moves in the $x z$ plane with angle $\rho_{0}$ when propagating along $z$, the amplitude for each $z$ shows increasing ellipticity, contributing differently to the total OAM decomposition of the spatial waveform of the two-photon state.

In conclusion, we have shown that the elucidation of the OAM correlations of entangled photons generated in SPDC can be addressed in two complementary scenarios, giving correspondingly different OAM correlations between the photons. One scenario considers the quantum amplitudes in the whole down-conversion cone, while the other scenario, experimentally relevant for quantum information protocols that make use of the OAM, pays attention to a small section of the down-conversion cone.

The results obtained here are of great importance for other configurations where entangled paired photons are generated. This is the case of pairs of photons generated through two-photon Raman transitions in electromagnetic induced transparency schemes [23]. Recently, the OAM correlations between stokes and anti-stokes photons has been measured in trapped Rubidium cold atoms in a counter-propagating nearly collinear geometry [24]. The measured correlations comply with the selection rule $m_{p}-m_{c}=m_{1}-m_{2}$, where $m_{c}$ refers to the OAM per photon of a laser control beam that counter propagates with the pump beam. The corresponding selection rule for counter propagating signal and idler photons [20] in SPDC would be $m_{p}=m_{1}-m_{2}$. We expect that a similar experiment in a highly noncollinear geometry, such a transverse emitting configuration 25], would yield strong departures from the selection rule $m_{p}-m_{c}=m_{1}-m_{2}$ measured for a nearly collinear geometry.

This work was supported by projects FIS2004-03556 and Consolider-Ingenio 2010 QOIT from Spain, by the European Commission (Qubit Applications (QAP), Contract No. 015848) and by the Generalitat de Catalunya.
[1] L. Allen, M. W. Beijersbergen, R. J. C. Spreeuw, and J. P. Woerdman, Phys. Rev. A 45, 8185 (1992).

[2] A. Mair, A. Vaziri, G. Weihs and A. Zeilinger, Nature 412, 313 (2001).

[3] G. Molina-Terriza, Juan P. Torres, and L. Torner, Phys. Rev. Lett. 88013601 (2002).

[4] J. P. Torres, A. Alexandrescu and L. Torner, Phys. Rev. A 68, 050301 (2003).

[5] G. Molina-Terriza, J. P. Torres and L. Turner., Nature Phys. 3, 305 (2007).

[6] A. Vaziri, G. Weihs and A. Zeilinger, Phys. Rev. Lett. 89, 240401 (2002).

[7] G. Molina-Terriza, A. Vaziri, R. Ursin and A. Zeilinger., Phys. Rev. Lett. 94, 040501 (2005).

[8] J. T. Barreiro, N. K. Langford, N. A. Peters and P. G. Kwiat, Phys. Rev. Lett. 95, 260501 (2005).

[9] S. P. Walborn, A. N. de Oliveira, R. S. Thebaldi and C. H. Monken, Phys. Rev. A 69, 023811 (2004).

[10] G. Molina-Terriza et al., Phys. Rev. Lett. 92, 167903 (2004).
[11] N. K. Langford et al., Phys. Rev. Lett. 93, 053601 (2004).

[12] A. R. Altman et al., Phys. Rev. Lett. 94, 123601 (2005).

[13] D. C. Burnham and D. L. Weinberg, Phys. Rev. Lett. 25, 84 (1970).

[14] G. Molina-Terriza, S. Minardi, Y. Deyanova, C. I. Osorio and M. Hendrych, Phys. Rev. A 72, 065802 (2005).

[15] H. H. Arnaut and G. A. Barbosa, , Phys. Rev. Lett. 85, 286 (2000).

[16] S. Franke-Arnold, S. M. Barnett, M. J. Padgett and L. Allen, Phys. Rev. A 65, 033823 (2002).

[17] J. P. Torres, G. Molina-Terriza and L. Torner, J. Opt. B 7, 235 (2005).

[18] A. Migdall, J. Opt. Soc. Am. B 14, 1093 (1997).

[19] D. N. Klyshko, Sov. Phys. JETP 28, 522 (1969).

[20] J. P. Torres, C. I. Osorio and L. Torner, Opt. Lett. 29, 1939 (2004).

[21] N. Bloembergen, "Conservation of angular momentum for optical processes in crystals", in Polarization, Matiere et rayonnement, Presses Universitaires, Paris, 109 (1969).

[22] M. H. Rubin, D.N. Klyshko, and Y.H. Shih, Phys. Rev. 
A 50, 5122 (1994).

[23] V. Balić et al., Phys. Rev. Lett. 94, 183601 (2005).

[24] R. Inoue et al., Phys. Rev. A 74, 053809 (2006).
[25] P. Kolchin et al., Phys. Rev. Lett. 97, 113602 (2006). 